\title{
Aspetti diagnostici ed epidemiologici delle peritoniti in pazienti sottoposti a dialisi peritoneale ambulatoriale continua (CAPD)
}

\author{
D. Crotti ${ }^{1}$, M.C. Medori ${ }^{1}$, C. Carobi $^{2}$, G.P. Gubbiotti ${ }^{2}$ \\ ${ }^{1}$ U.O. di Analisi Chimico-cliniche e Microbiologiche, \\ ${ }^{2}$ U.O. di Nefrologia e Dialisi, \\ Ospedale R. Silvestrini, 06100 S. Sisto, Perugia
}

a dialisi peritoneale ambulatoriale continua (CAPD), valida alternativa all'emodialisi in pazienti con insufficienza renale, presenta peraltro l'inconveniente di complicanze locali di natura infettiva, per la cui diagnostica non sono ancora state standardizzate adeguate tecniche o metodologie specifiche (1).

La necessità di protocolli idonei è peraltro quanto mai reale (2), sia per minimizzare rischiose manovre strumentali, sia per agevolare, omogeneizzare ed ottimizzare i criteri operativi microbiologici (3), sia, infine, per garantire un'efficienza diagnostica altrimenti aleatoria o fuorviante $(1,4,5)$.

Abbiamo pertanto verificato la bontà di un iter di laboratorio che potesse da un lato garantire la più ampia possibile individuazione eziologica di peritoniti in pazienti sottoposti a CAPD $(6,7)$, fornendoci così un quadro realistico dell'epidemiologia di tali infezioni (2), e dall'altro offrire o suggerire indicazioni per reimpostare un razionale protocollo diagnostico, sicuro, concreto e riproducibile.

\section{Materiali e metodi}

Tutti i campioni di liquido peritoneale prelevati nei pazienti con sospetta peritonite (dolori addominali e/o liquido peri- toneale torbido), venivano inviati in laboratorio, sulla base di un protocollo operativo concordato in precedenza (Tab. I).

Qualora, per motivi contingenti, non era possibile l'immediato invio in laboratorio, le brodocolture venivano tenute in termostato e campione di liquido peritoneale in frigorifero in reparto.

Sono stati esaminati 314 liquidi peritoneali per sospetto di peritonite (o, comunque, per un controllo volto ad escludere la stessa).

I campioni sono stati suddivisi in 2 gruppi: 216 per un primo gruppo, relativo al periodo compreso tra l'inizio del secondo semestre del 1990 e la fine del primo semestre del 1992 (I periodo), 98 per un secondo gruppo, relativo al periodo compreso dall'inizio del secondo semestre 1992 al 31 ottobre 1993 (II periodo). L'unica differenza tra $\mathrm{i}$ due periodi fu nell'aver utilizzato il brodo Liquoid BHI con lisante nel II periodo rispetto all'uso di brodo Liquoid BHI senza lisante nel I. Per tutti i campioni (con alcune eccezioni) sono state seguite le indicazioni riportate nella Tabella I.

\section{Risultati e discussione}

Abbiamo osservato 90 casi di peritoniti in CAPD, pari al $28.7 \%$ del totale dei campioni esaminati. I casi positivi sono stati $60(28 \%)$ nel I periodo e $30(31 \%)$ nel secondo. Nelle Tabelle II-VI sono riportati nel dettaglio i risultati ottenuti. Accanto ad una prevalenza di gram positivi $(63 \%)$, si è assistito, nel II periodo, ad un incremento nelle eziologie da bastoncelli gram negativi (43\%, rispetto ad un $15 \%$ nel I periodo), con un corrispettivo calo di gram positivi (dal $70 \%$ al $50 \%$ ). I fomiceti e corineformi (5-7) sono stati osservati esclusivamente nel I periodo. Non sono mai stati isolati micobatteri o micoplasmi urogenitali.

Sono stati inoltre valutati il rapporto tra numero di globuli bianchi/ $/ \mathrm{mm}^{3}$ (conteggio automatico dei GB con Coulter STKS) ed esito colturale, il rapporto tra colorazione iniziale secondo Gram sul sedimento del liquido peritoneale ed esito colturale, il rapporto tra coltura in brodo (con semina di 10-15 ml di liquido peritoneale in flacone Liquoid direttamente al letto del malato e sottocolture su agar non selettivo quando positive $o$ terminali alla cieca al $7^{\circ}$ giorno) e coltura diretta di terreni agarizzati non selettivi (semina del sedimento del liquido peritoneale in laboratorio su agar sangue ed agar cioccolato).

Tali rapporti sono stati condotti su un campione significativo della popolazione reclutata nel II periodo considerato, ed erano finalizzate alla verifica dell'utilità 
TABELLA I - SCHEMATIZZAZIONE DEL PROTOCOLLO OPERATIVO INIZIALE ADOTTATO

A) inoculo diretto (al letto del malato) di flacone con brodo (Liquoid BHI/Liquoid BHI con lisante: $7 \mathrm{gg}$ a $37^{\circ} \mathrm{C}$ )

B) inoculo diretto (al letto del malato) di flacone Liquoid THIO (saltuariamente: $7 \mathrm{gg}$ a $\left.37^{\circ} \mathrm{C}\right)$

C) 1 contenitore sterile con $50 \mathrm{cc}$ di liq. peritoneale:

1) valutazione macroscopica della torbidità (in Lab.)

2) conteggio automatico GB (in lab.)

3) col. sec. Gram dal sedimento (centrifugazione di $10 \mathrm{cc}$ a $2000 \mathrm{~g} \mathrm{x} 15 \mathrm{~m}$ )

4) in base al conteggio dei GB:

a) se $100 / \mathrm{m}^{3}$ :

- col. sec. Gram

- agar sangue $\left(\mathrm{O}_{2}, 37^{\circ} \mathrm{C}, 72 \mathrm{~h}\right)$

- agar cioccolato $\left(\mathrm{CO}_{2}, 37^{\circ} \mathrm{C}, 72 \mathrm{~h}\right)$

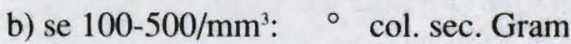

- col. MGG

- agar sangue (come sopra)

- agar cioccolato (come sopra)

- agar cioccolato (microaerofilia, $37^{\circ} \mathrm{C}, 72 \mathrm{~h}$ )

- ricerca micoplasmi urogenitali

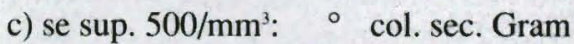

- col. MGG

- acridine-orange (in fluorescenza)

- agar sangue ed agar cioccolato (come sopra)

- agar cioccolato in microaerofilia (come sopra)

- agar Schaedler $\left(-\mathrm{O}_{2}, 37^{\circ} \mathrm{C}, 72 \mathrm{~h}\right)$

- agar Sabouraud $\left(\mathrm{O}_{2}, 37^{\circ} \mathrm{C}, 5 \mathrm{gg}\right)$

- ricerca micoplasmi urogenitali

d) conservazione di $30 \mathrm{ml}$ di liquido peritoneale per $3 \mathrm{gg} \mathrm{a} 4^{\circ} \mathrm{C}$

e) nel caso di negatività microbiologica al $3^{\circ}$ giorno (per i punti b e c):

- colorazione sec. Ziehl-Neelseen

- semina su IUTM $\left(60 \mathrm{gg}\right.$ a $\left.37^{\circ} \mathrm{C}\right)$ (da sedimento spontaneo centrifugato: $15 \mathrm{~m}$ a 2000 giri)

5) solo per conteggi superiori a $100 \mathrm{~GB} / \mathrm{mm}^{3}$ (punti b e c di 4): inoculo di $10-20 \mathrm{ml}$ di liquido peritoneale in provette FTM $135-\mathrm{C}$ (incubazione a $37^{\circ} \mathrm{C}$ per 3 giorni) di alcuni criteri prediagnostici o diagnostici al riguardo.

In 20 casi di peritoniti ad eziologia accertata il conteggio dei GB (oltre il 90\% era rappresentato sempre da polimorfonucleati neutrofili) è risultato superiore a $100 / \mathrm{mm}^{3}$ in tutti i casi, con il $95 \%$ superiore a $500 / \mathrm{mm}^{3}$. All'interno di un gruppo di 76 campioni negativi, il conteggio dei GB è risultato inferiore a $100 / \mathrm{mm}^{3}$ nell' $86 \%$ degli stessi, inferiore a $500 / \mathrm{mm}^{3}$ nel $97 \%$; in un solo caso $(3 \%)$ il conteggio fu superiore a $500 / \mathrm{mm}^{3}$. Ne consegue la opportunità di non processare campioni di liquidi peritoneali (limpidi) con conteggi inferiori a $100 \mathrm{~GB} / \mathrm{mm}^{3}$. La colorazione secondo Gram condotta sul sedimento del liquido perioneale ha fornito indicazioni preliminari riguardo una potenziale eziologia microbica soltanto nel 20\% dei casi positivi studiati (4 su 20). Ne deriva la pressoché totale inutilità di tale colorazione preliminare; ciò è verosimilmente collegato alla frequente bassa concentrazione batterica nella maggior parte delle peritoniti dei pazienti sottoposti a CAPD (1).

Per quanto concerne la colorazione tra crescita diretta su terreni agarizzati e crescita in terreni liquidi, si è osservata una correlazione positiva nell' $87 \%$ dei casi. In 4 su 30 campioni positivi studiati soltanto la coltura in brodo permise un isolamento microbico. Trattavasi di 4 casi ad eziologia variata, con conteggi di GB sempre superiori a $400 / \mathrm{mm}^{3}$. Discutibile potrebbe pertanto apparire l'utilità di utilizzare sia terreni liquidi che terreni agarizzati. Questo anche in considerazione del fatto che i tempi diagnostici risultano analoghi. Nel nostro studio, infatti, in quasi tutti i casi di concordanza positiva, mentre la torbidità del brodo si osservava dopo 24-48 ore, le colonie si sviluppavano sulle piastre di agar sangue/agar cioccolato soltanto dopo 48-72 ore. Considerando la necessità della sicurezza di una subcoltura in piastra dopo crescita in brodo, i tempi finali diagnostici sono risultati pressoché del tutto sovrapponibili. $\mathrm{Va}$ inoltre riferito che nessuna informazione in più è stata fornita dall'uso combinato di agar sangue (in anaerobiosi) e di agar cioccolato (in capnofilia).

\section{Conclusioni}

Le conclusioni che si possono trarre da questo studio sono sicuramente prope- 
TABELLA II - RISULTATI COMPLESSIVI: DATI GENERALI

\begin{tabular}{lccc}
\hline & $90-93$ & $90-92$ & $92-93$ \\
\hline Totali positivi & 90 & 60 & 30 \\
Gram positivi & 57 & 42 & 15 \\
Gram negativi & $63 \%$ & $70 \%$ & $50 \%$ \\
& 22 & 9 & 13 \\
Anaerobi stretti & $25 \%$ & $15 \%$ & 1 \\
& 3 & 2 & $3 \%$ \\
Lieviti & $3 \%$ & $3 \%$ & 2 \\
Muffe & 5 & $5 \%$ & $7 \%$ \\
& $5 \%$ & 4 & 0 \\
\hline
\end{tabular}

ND: 2 associazioni (S. marcescens - C. albicans)

TABELLA III - RISULTATI COMPLESSIVI: EZIOLOGIE DA GRAM POSITIVI

\begin{tabular}{lccc} 
& Totali & I periodo & II periodo \\
\hline Stafilococchi & 47 & 35 & 12 \\
Streptococchi & $82 \%$ & $83 \%$ & $80 \%$ \\
& 6 & 3 & 3 \\
Corineformi & $11 \%$ & $7 \%$ & $20 \%$ \\
& 4 & 4 & 0 \\
\hline Totali & $7 \%$ & $42 \%$ & - \\
\hline
\end{tabular}

TABELLA IV - RISULTATI COMPLESSIVI: EZIOLOGIE SOSTENUTE DA COCCHI E BASTONCELLI GRAM POSITIVI

\begin{tabular}{|c|c|c|c|c|c|c|}
\hline \multirow[b]{2}{*}{ S. aureus } & \multicolumn{2}{|c|}{ Totali } & \multicolumn{2}{|c|}{ I periodo } & \multicolumn{2}{|c|}{ II periodo } \\
\hline & 20 & $35 \%$ & 14 & $33 \%$ & 6 & $40 \%$ \\
\hline S. epidermidis & 22 & $39 \%$ & 19 & $45 \%$ & 3 & $20 \%$ \\
\hline Staphylococcus sp. & 5 & $8 \%$ & 2 & $5 \%$ & 3 & $20 \%$ \\
\hline S. sanguis & 3 & & 1 & & 2 & \\
\hline S. mitis & 2 & $11 \%$ & 1 & $7 \%$ & 1 & $20 \%$ \\
\hline G. morbillorum & 1 & & 1 & & 0 & \\
\hline C. jeikeium & 1 & & 1 & & 0 & 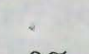 \\
\hline R. equi & 1 & $7 \%$ & 1 & $10 \%$ & 0 & $0 \%$ \\
\hline Brevibacterium sp. & 2 & & 2 & & 0 & \\
\hline
\end{tabular}


TABELLA V - RISULTATI COMPLESSIVI: EZIOLOGIE SOSTENUTE DA BASTONCELLI GRAM NEGATIVI

\begin{tabular}{|c|c|c|c|c|c|c|}
\hline \multirow[b]{2}{*}{ Enterobatteri } & \multicolumn{2}{|c|}{ Totali } & \multicolumn{2}{|c|}{ I periodo } & \multicolumn{2}{|c|}{ II periodo } \\
\hline & 14 & $64 \%$ & 5 & $56 \%$ & 9 & $69 \%$ \\
\hline E. coli & 8 & & 3 & & 5 & \\
\hline S. marcescens & 3 & & 1 & & 2 & \\
\hline P. mirabilis & 1 & & 1 & & 0 & \\
\hline E. cloacae & 1 & & 0 & & 1 & \\
\hline K. oxytoca & 1 & & 0 & & 1 & \\
\hline Glucosio non fermentanti & 8 & $36 \%$ & 4 & $44 \%$ & 4 & $31 \%$ \\
\hline P. aeruginosa & 4 & & 1 & & 3 & \\
\hline P. fluorescens & 1 & & 1 & & 0 & \\
\hline P. vesciularis & 2 & & 1 & & 1 & \\
\hline Neisseria sp. & 1 & & 1 & & 0 & \\
\hline
\end{tabular}

TABELLA VI - RISULTATI COMPLESSIVI: EZIOLOGIE SOSTENUTE DA ANAEROBI OBBLIGATI E FUNGHI

\begin{tabular}{lccc}
\hline & Totali & I periodo & II periodo \\
\hline Anaerobi stretti & 3 & 2 & 1 \\
B. fragilis & 1 & 1 & 0 \\
P. acnes & 1 & 1 & 0 \\
Clostridium sp. & 1 & 0 & 1 \\
Funghi & 7 & 5 & 2 \\
C. albicans & 3 & 1 & 2 \\
P. varioti & 1 & 1 & 0 \\
M. racemosus & 1 & 1 & 0 \\
A. candidus & 1 & 1 & 0 \\
Ifomicete non identificato & 1 & 1 & 0 \\
\hline
\end{tabular}

deutiche per una migliore razionalizzazione diagnostica. Innanzitutto è opportuno processare microbiologicamente esclusivamente i campioni con conteggi superiori a $100 \mathrm{~GB} / \mathrm{mm}^{3}$.

Per quanto riguarda i campioni liquidi o apparentemente tali si devono utilizzare esclusivamente terreni liquidi (raccolti al letto del malato e subito inviati in laboratorio). Per i campioni torbidi con conteggi di GB superiori a $500 / \mathrm{mm}^{3}$ potrebbe essere efficace procedere all'utilizzazione anche di piastre agarizzate inse- menzate in prima giornata con il sedimento del liquido peritoneale del paziente, purché si scelgano adeguati terreni non selettivi ed adeguate temperature ed atmosfere di incubazione.

Potrebbe così essere opportuno affiancare un agar cioccolato in microaerofilia all'agar sangue in normale tensione di ossigeno, optando sempre per la temperatura di $37^{\circ} \mathrm{C}$ ma prorogando a 3 giorni i tempi di incubazione.

La colorazione secondo Gram potrebbe essere eseguita esclusivamente in liquidi francamente torbidi, con conteggi elevati di GB, eventualmente prolungando i tempi ed i giri di centrifugazione. I brodi debbono essere osservati quotidianamente, con sottocolture immediate qualora si rilevi o si sospetti una positività. Laddove si utilizzino brodi con lisante (e potrebbero essere più indicati) l'utilità di affiancare piastre dirette è indubbia. In ogni caso è doverosa una sottocoltura anche alla cieca al $7^{\circ}$ giorno, con prolungamento dell'incubazione della piastra finale (o delle piastre finali) fino a 3 
TABELLA VII - PROTOCOLLO OPERATIVO RIPROPOSTO PER LA DIAGNOSTICA DELLE PERITONITI IN PAZIENTI SOTTOPOSTI A CAPD

PROTOCOLLO OPERATIVO

Invio liquido peritoneale in laboratorio:

A) limpido/conteggio GB inf.re a $500 / \mathrm{mm}^{3}$ :

1) flacone come per emocoltura (inoculo al letto del malato)

B) limpido/conteggio GB $\geq 500 / \mathrm{mm}^{3}$ :

1) come in 1) di A)

2) al $3^{\circ}$ giorno, se negativo, sottocolture alla cieca su:

- agar sangue (aerobiosi)

- agar cioccolato (microaerofilia)

- agar Schaedler (anaerobiosi)

a $37^{\circ} \mathrm{C} \times 3 \mathrm{gg}$

3) al $6^{\circ}$ giorno, se negativo, sottocoltura in:

- IUTM (secondo le consuete indicazioni)

N.B.: il liquido peritoneale va conservato a $4^{\circ} \mathrm{C}$ per $6 \mathrm{gg}$

C) torbido / conteggio GB inf.re a $500 / \mathrm{mm}^{3}$ :

1) come in 1) di A)

D) torbido / conteggio GB $\geq 500 / \mathrm{mm}^{3}$ :

1) come in 1) di A)

2) eseguire sedimentazione ( $3.000 \mathrm{~g} \mathrm{x} 30 \mathrm{~min})$ :

- colorazione sec. Gram:

a) se negativa: come punto 2) e 3) di B)

b) se positiva: comportarsi in base al caso singolo

\section{BIBLIOGRAFIA}

1. von Graevenitz A, Amsterdam D. Microbiological aspects of peritonitis associated with continuous ambulatory peritoneal dialysis. Clin Microb Rev 1992; 5: 36-48.

2. Crotti D, D'Annibale ML, Del Sante M, Fonzo G, Vinti AM. Peritoniti in CAPD: diagnosi microbiologica. Microb Med 1993; 8: 202-3.

3. Doyle PW, Crichton EP, Mathias RG, Werb R. Clinical and microbiological evaluation of four colture methods for the diagnosis of peritonitis in patients on continuous ambulatory peritoneal dialysis. J Clin Microbiol 1989; 27: 1206-9.

4. von Graevenitz A, Pfyffer GE, Ickett MJ, Weaver RE, Wuest J. Isolation of an unclassified non-fermentative gram-negative rod from a patient on continuous ambulatory peritoneal dialysis. Eur J Clin $\mathrm{Mi}$ crobiol Infect Dis 1993; 12: 568-70.

5. Wuest J, Lanzendorfer LH, von Graevenitz A, Gloor HJ, Schmid B. Peritonitis caused by Actinomadura madurae in a patient on CAPD. Eur J Clin Microbiol Infect Dis 1990; 9: 700-1. giorni. In Tabella VII riproponiamo un protocollo diagnostico che dovrebbe essere più razionale ed altrettanto efficace, alla luce dei risultati scaturiti dallo studio fatto.

Per concludere, riteniamo che la variabilità eziologica delle peritoniti in pazienti CAPD, anche da noi osservata, in gran parte è conseguente alla assoluta peculiarità della dialisi peritoneale continua stessa. Un monitoraggio continuo ed un controllo continuo sono peraltro e pertanto doverosi al riguardo.
6. Gruner E, Pfyffer GE, von Graivenitz A. Characterization of Brevibacterium sp. from clinical specimens. J Clin Microbiol 1993; 31: 1408-12.

7. Marzec A, Heron LG, Pritchard RC, et al. Paecilomyces variotii in peritoneal dialysate. J Clin Microbiol 1993; 31: 2392-5. 